\title{
Pseudoaneurysm at the origin of the left subclavian artery following type A interrupted aortic arch repair in adulthood, London, United Kingdom
}

\author{
Georgios Belitsis, MD, Jonathan R. Finch, MD, PhD, Darryl F. Shore, MD, and Ulrich P. Rosendahl, MD, \\ London, United Kingdom
}

\footnotetext{
From the Department of Cardiothoracic Surgery and Transplantation, Royal Brompton and Harefield Hospitals, London, United Kingdom.

Disclosures: Authors have nothing to disclose with regard to commercial support.

Received for publication Aug 28, 2015; accepted for publication Sept 3, 2015; available ahead of print Oct 20, 2015.

Address for reprints: Georgios Belitsis, MD, Department of Cardiothoracic Surgery and Transplantation, Harefield Hospital, Hill End Rd, Harefield, Middlesex UB9 6JH, United Kingdom (E-mail: gbelitsis@ gmail.com). J Thorac Cardiovasc Surg 2016;151:e17-9 $0022-5223 / \$ 36.00$

Copyright (c) 2016 by The American Association for Thoracic Surgery http://dx.doi.org/10.1016/j.jtcvs.2015.09.022
}

We present the case of a male adult aged 44 years who has congenital heart disease. Congenital pathology involved left-sided interrupted aortic arch (IAA) type A, situs solitus, D looping of the heart, and normal arrangement of great arteries at their origin. No intracardiac or extracardiac shunts were noted. The left ventricle volumes were normal and the aortic valve consisted of 3 cusps. The epiaortic branches arising in the orthotopic fashion were (from proximal to distal): bracheocephalic trunk, left common carotid artery (CCa), and left subclavian artery (SCa). The ductus arteriosus was obliterated. There was no intracardiac pathology. ${ }^{1,2}$

\section{CLINICAL SUMMARY}

During his 22nd year of life the patient underwent surgery for the IAA through a left posteriolateral thoracotomy. ${ }^{3}$ Intraoperatively, the left $\mathrm{SCa}$ appeared to be 3 inches away from the most superior part of the descending thoracic aorta. ${ }^{4}$ A $20-\mathrm{mm}$ vascular synthetic conduit was anastomosed from the left subclavian artery to the proximal descending aorta. Routine follow-up magnetic resonance imaging suggested aneurysmatous changes on the proximal anastomosis on the left SCa. Computed tomography aortogram confirmed pseudoaneurysm near the anastomosis site between the left SCa and vascular conduit (Figure 1,A). The pseudoaneurysm measured $40 \mathrm{~mm}$. Surgery was planned. With the patient in a supine position the left $\mathrm{CCa}$ was anastomosed to the distal left $\mathrm{SCa}$ with an 8-mm synthetic vascular graft. An 8-mm side arm was there to be used for arterial return of the cardiopulmonary bypass circuit. The patient was turned on his left lateral side, maintaining access to the left femoral region in case cardiopulmonary bypass would be necessary. Then an extended redo antero/posterolateral thoracotomy through the fourth intercostal space was performed. The dissection was extremely difficult due to dense adhesions of the lung and the

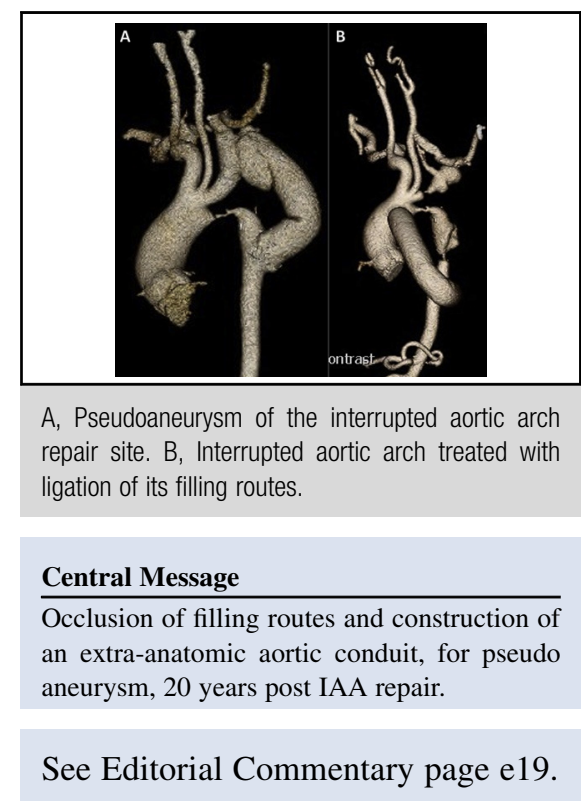

aneurysm to the thoracic wall. The superior lobe of the left lung was fully adherent to the aneurysm. The adhesions around the aortic arch were dissected. The bracheocephalic trunk, the left $\mathrm{CCa}$, and the left $\mathrm{SCa}$ were visualized. It was determined at the time that there was no safe way to dissect the pseudoaneurysm from the surrounding adhesions, followed by aneurysmectomy and end-to-end orthotropic reconstruction of the IAA. The use of an extra-anatomic conduit from ascending to descending aorta came into the plan, with ligation of the aneurism major filling paths (Figure 1, A).

Systemic heparinization took place (target activated clotting time 300 seconds). A $26-\mathrm{mm}$ synthetic vascular graft was anastomosed end to side to the distal ascending aorta using partial vascular clamps. The graft was passed anteriorly to the root of the left lung and anastomosed end to side to the distal descending thoracic aorta, also using a partial aortic clamp. The left SCa was ligated at its origin. The proximal descending thoracic aorta was ligated just distal to the insertion of the previously placed conduit. The extrathoracic part of the left SCa was ligated proximal to the insertion of the graft and distal to the origin of the left vertebral artery. The side arm of the vascular conduit was flashed so the clot was washed out, and it was ligated very near its origin. The patient was discharged from our 

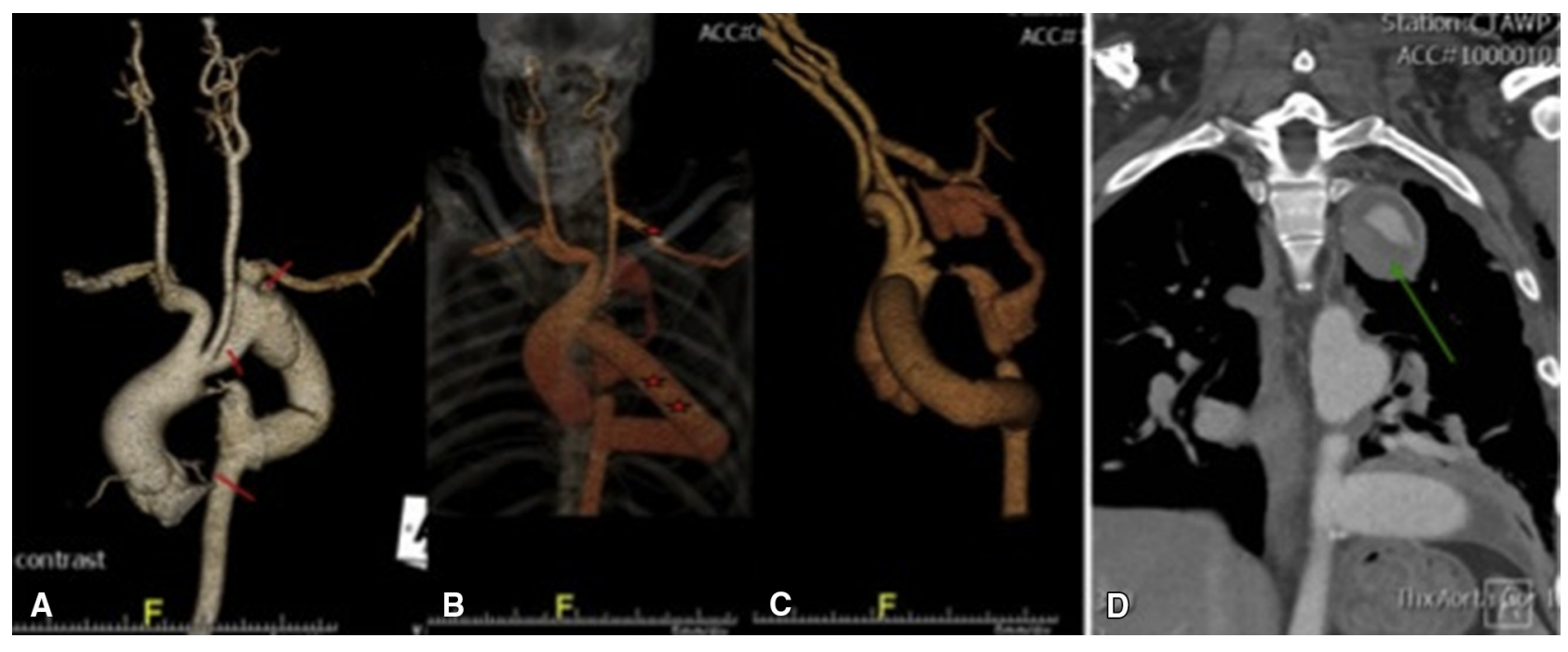

FIGURE 1. A, Preoperative aortogram. The red lines mark ligation points at surgery. B, Two weeks postoperative aortogram (anterio posterior view) with single red star marking shunt between the left common corotid artery and the left subclavian artery; double red star marks ascending to descending aorta extra anatomic conduit. C, Lateral view demonstrating the partially thrombosed original conduit for comparison with the preoperative image. D, Coronal view of 2 weeks postoperative computed tomography angiogram (see Figure 2) showing partially thrombosed pseudoaneurysm (green arrow).

intensive care unit on postoperative day 2 and from the hospital 2 weeks after his operation. He experienced a recurrent laryngeal nerve injury. The postoperative computed tomography angiogram (on the second week postoperation) confirmed good flow in the extra-anatomic ascending to descending conduit. The original conduit and the aneurysm site had already started to thrombose
(Figure 1, B-D). It was still getting filled mainly from the left vertebral artery. The patient was seen 4 months after the operation in our outpatient clinic. Recovery was spectacular. Had reached the point of cycling more than $20 \mathrm{~km} / \mathrm{d}$ and swimming more than $3 \mathrm{~h} / \mathrm{d}$. Recurrent laryngeal nerve injury symptoms have markedly recovered. On repeat computed tomography aortogram (Figure 2)
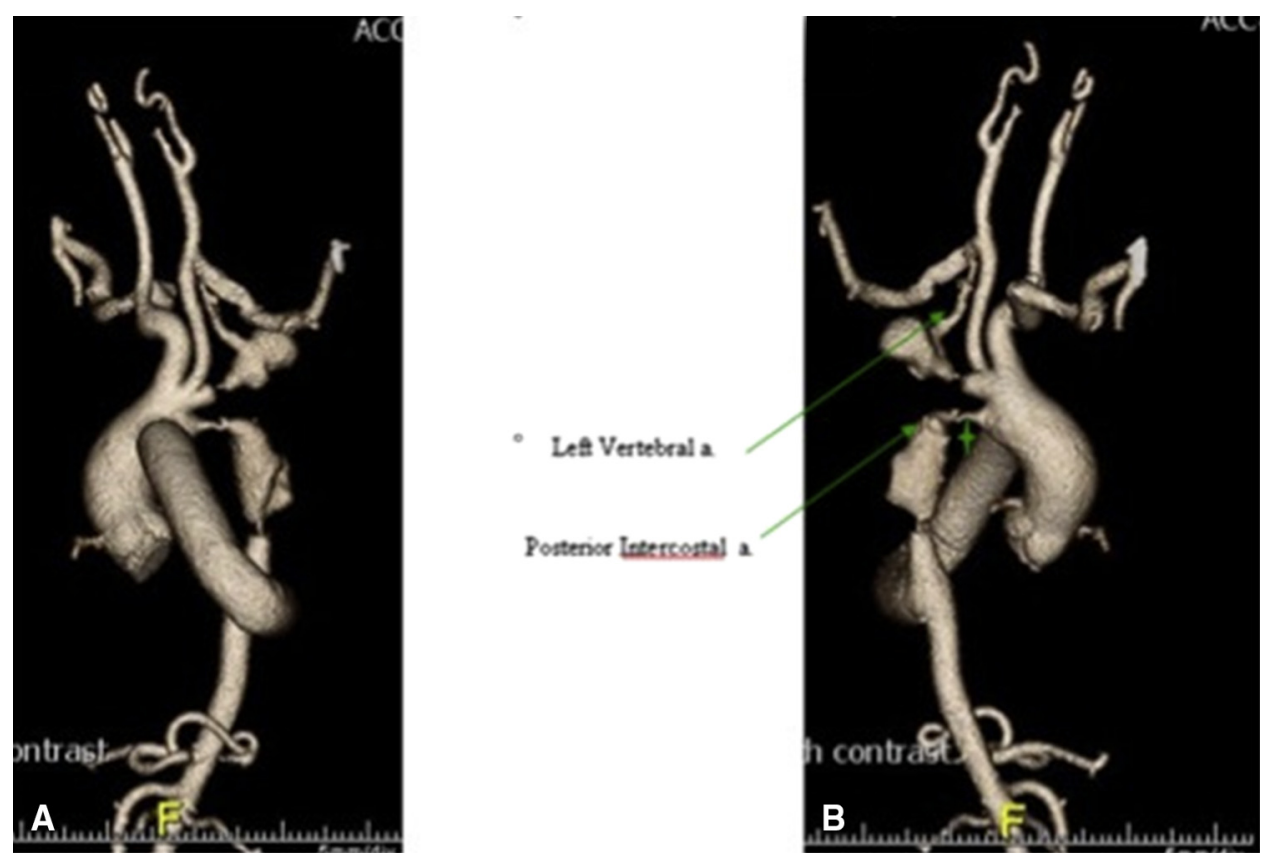

FIGURE 2. A, Lateral view of the computed tomography aortogram 4 months postoperation showing further thrombosis of the original conduit. B, Posterior anterior view of computed tomography aortogram 4 months postoperation showing the original conduit filling mainly from the left vertebral artery superiorly and from the interconstal arteries inferiorly, the hypoplastic/aplastic isthmus appears recanalizing (green asterisk). 
4 months after the operation the extra-anatomic ascending to descending conduit remained widely patent. There has been further thrombosis of the original descending vascular conduit and the adjacent false aneurysm. Two separate remaining collections now fill with contrast medium: a superior collection that fills predominantly from the left vertebral artery but also with a narrow channel from the vestigial isthmus, and an inferior collection that fills by a very narrow channel from the descending aorta, possibly from interconstals back-bleeding in the very proximal de- scending aorta that was still connected to the conduit. Routine computed tomography follow up is planned.

\section{References}

1. Gatzoulis MA, Swan L, Therrien J, Pantely GA. Adult congenital heart disease: a practical guide. Malden, MA: Blackwell Books; 2005.

2. Ruiz Villabor MC, DeBalderrama DP. Lopez y Lopez J, Castelanos M. Complete interruption of the aortic arch. Am J Cardiol. 1961;8:664-9.

3. Reardon MJ, Hallman GL, Cooley DA. Interrupted aortic arch: brief review and summary of an eighteen-year experience. Tex Heart Inst J. 1984;11:250-9.

4. Gohcebay TM. Complete interruption of the aorta at the arch. Am J Roentgenol Radium Ther Nucl Med. 1972;114:362-70.

\title{
EDITORIAL COMMENTARY
}

\section{Complex congenital aortic arch disease: The need for mandatory long-term follow-up}

\author{
Harold M. Burkhart, MD, Jess L. Thompson, MD, and Sabrina D. Phillips, MD
}

\footnotetext{
From the Division of Cardiovascular and Thoracic Surgery, University of Oklahoma Health Sciences Center, Oklahoma City, Okla.

Disclosures: Authors have nothing to disclose with regard to commercial support.

Received for publication Sept 21, 2015; accepted for publication Sept 25, 2015; available ahead of print Oct 23, 2015.

Address for reprints: Harold M. Burkhart, MD, Division of Cardiovascular and Thoracic Surgery, University of Oklahoma Health Sciences Center, PO Box 26901, WP-2230, Oklahoma City, OK 73105 (E-mail: Harold-burkhart@ouhsc.edu).

J Thorac Cardiovasc Surg 2016;151:e19-20

$0022-5223 / \$ 36.00$

Copyright (C) 2016 by The American Association for Thoracic Surgery http://dx.doi.org/10.1016/j.jtcvs.2015.09.113
}

Patients with coarctation and interrupted aortic arch comprise a group of adults with congenital heart disease at increased risk for life-threatening, lifelong postoperative sequelae. ${ }^{1,2}$ The risks after aortic reconstruction are well described and include recoarctation or stenosis as well as aneurysm and pseudoaneurysm formation. Placing these patients at further risk is the fact that the complication of aneurysm or pseudoaneurysm is often asymptomatic until aortic catastrophe occurs. Other issues, including the risk of hypertension, heart disease, and decreased survival, add to the challenges of providing appropriate long-term care to these complex patients. Unfortunately, as these patients age, they are often no longer receiving follow-up because they are erroneously considered to have had a simple congenital heart disease that was "cured" with surgery.

Belitsis and colleagues ${ }^{3}$ present a case of interrupted aortic arch, discovered in adulthood, repaired through a left thoracotomy with a left subclavian artery-descending aorta interposition graft. Two decades later, computed

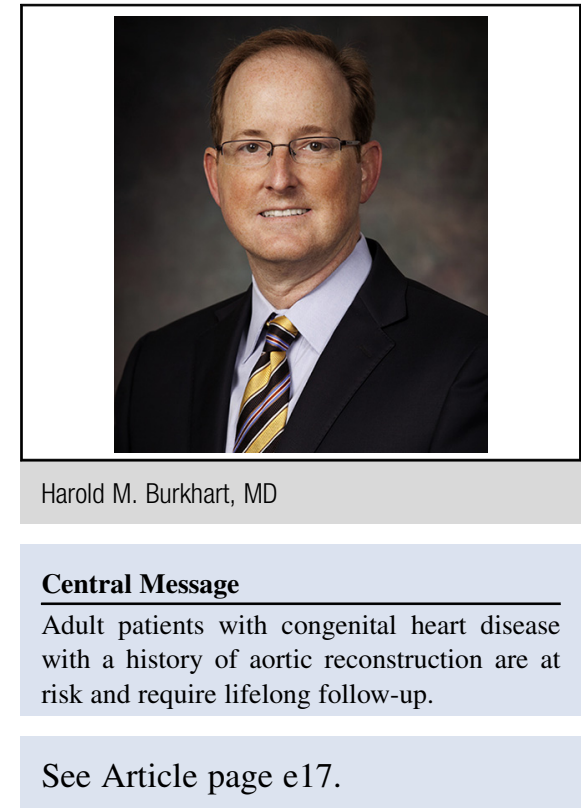

tomography revealed a 4-cm pseudoaneurysm at the left subclavian anastomosis. After a left subclavian-left common carotid artery bypass was performed, the left side of the chest was reopened to excise the pseudoaneurysm and reconstruct the aorta. Not surprisingly, the dissection proved to be difficult, resulting in the surgeon's aborting the original plan and performing an ascendingdescending aortic bypass with near exclusion of the aneurysmal aortic segment. Apart from a recurrent 